OPEN ACCESS

Edited by:

Irena Levitan,

University of Illinois at Chicago,

United States

Reviewed by:

Raghu P. Kataru,

Memorial Sloan Kettering Cancer

Center, United States

Kishore K. Wary,

University of Illinois at Chicago,

United States

*Correspondence:

Edward M. Schwarz

Edward_Schwarz@

URMC.Rochester.edu

Specialty section:

This article was submitted to

Vascular Physiology,

a section of the journal

Frontiers in Physiology

Received: 21 July 2021 Accepted: 01 September 2021 Published: 27 September 2021

Citation:

Scallan JP, Bouta EM, Rahimi H, Kenney HM, Ritchlin CT, Davis MJ and Schwarz EM (2021) Ex vivo

Demonstration of Functional Deficiencies in Popliteal Lymphatic Vessels From TNF-Transgenic Mice

With Inflammatory Arthritis.

Front. Physiol. 12:745096. doi: 10.3389/fphys.2021.745096

\section{Ex vivo Demonstration of Functional Deficiencies in Popliteal Lymphatic Vessels From TNF-Transgenic Mice With Inflammatory Arthritis}

\author{
Joshua P. Scallan 1,2, Echoe M. Bouta ${ }^{3,4}$, Homaira Rahimi3,5,6, H. Mark Kenney ${ }^{3,6}$, \\ Christopher T. Ritchlin ${ }^{3,7}$, Michael J. Davis ${ }^{1}$ and Edward M. Schwarz ${ }^{3,4,6,8 *}$
}

'Department of Medical Pharmacology and Physiology, University of Missouri, Columbia, MO, United States, ${ }^{2}$ Department of Molecular Pharmacology and Physiology, Morsani College of Medicine, University of South Florida, Tampa, FL, United States, ${ }^{3}$ Center for Musculoskeletal Research, Rochester, NY, United States, ${ }^{4}$ Department of Biomedical Engineering, Rochester, MI, United States, ${ }^{5}$ Department of Pediatrics, Rochester, NY, United States, ${ }^{6}$ Department of Pathology and Laboratory Medicine, Rochester, NY, United States, ' Division of Allergy, Immunology, Rheumatology, Department of Medicine, Rochester, NY, United States, ${ }^{8}$ Department of Orthopaedics, University of Rochester School of Medicine and Dentistry, Rochester, NY, United States

Background: Recent studies demonstrated lymphangiogenesis and expansion of draining lymph nodes during chronic inflammatory arthritis, and lymphatic dysfunction associated with collapse of draining lymph nodes in rheumatoid arthritis (RA) patients and TNF-transgenic (TNF-Tg) mice experiencing arthritic flare. As the intrinsic differences between lymphatic vessels afferent to healthy, expanding, and collapsed draining lymph nodes are unknown, we characterized the ex vivo behavior of popliteal lymphatic vessels (PLVs) from WT and TNF-Tg mice. We also interrogated the mechanisms of lymphatic dysfunction through inhibition of nitric oxide synthase (NOS).

Methods: Popliteal lymph nodes (PLNs) in TNF-Tg mice were phenotyped as Expanding or Collapsed by in vivo ultrasound and age-matched to WT littermate controls. The PLVs were harvested and cannulated for ex vivo functional analysis over a relatively wide range of hydrostatic pressures $\left(0.5-10 \mathrm{cmH}_{2} \mathrm{O}\right)$ to quantify the end diastolic diameter (EDD), tone, amplitude (AMP), ejection fraction (EF), contraction frequency (FREQ), and fractional pump flow (FPF) with or without NOS inhibitors Data were analyzed using repeated measures two-way ANOVA with Bonferroni's post hoc test.

Results: Real time videos of the cannulated PLVs demonstrated the predicted phenotypes of robust vs. weak contractions of the WT vs. TNF-Tg PLV, respectively. Quantitative analyses confirmed that TNF-Tg PLVs had significantly decreased AMP, EF, and FPF vs. WT $(p<0.05)$. EF and FPF were recovered by NOS inhibition, while the reduction in AMP was NOS independent. No differences in EDD, tone, or FREQ were observed between WT and TNF-Tg PLVs, nor between Expanding vs. Collapsed PLVs. 
Conclusion: These findings support the concept that chronic inflammatory arthritis leads to NOS dependent and independent draining lymphatic vessel dysfunction that exacerbates disease, and may trigger arthritic flare due to decreased egress of inflammatory cells and soluble factors from affected joints.

Keywords: inflammatory arthritis, lymphatic vessels, ex vivo analysis, mouse models, vessel contraction

\section{INTRODUCTION}

Rheumatoid arthritis (RA) is an inflammatory joint disease that affects $0.5-1 \%$ of the population (Firestein, 2003; Rudan et al., 2015). While there have been major advances in our understanding of RA pathogenesis, significant unmet clinical needs remain for many patients who are refractory to available treatments (Weinblatt et al., 1999, 2003). Although specific autoantibodies are diagnostic of RA, it is now broadly accepted that environmental and epigenetic factors are also critical in the development and pathogenesis of joint disease (Firestein, 2014). Additionally, the mechanisms of inflammatory joint disease in RA patients who do not have detectable levels of these autoantibodies (seronegative RA) are poorly understood (Pratt and Isaacs, 2014). Thus, research is needed to elucidate nonautoimmune etiologies of RA.

Another major challenge for RA patients and caregivers is the tendency of the disease to flare, and the relative refractory nature of persistent disease despite aggressive therapy (Firestein, 2014). Although autoimmune mechanisms underlie the development of RA, the pathways that trigger flare are not well understood, and alternative pathways may contribute to disease exacerbation (Bouta et al., 2018). Although recent gene expression studies have identified potential roles of immature $\mathrm{IgD}^{+} \mathrm{B}$-cells, circulating $\mathrm{CD} 45^{-} / \mathrm{CD} 31^{-} / \mathrm{PDPN}^{+}$ pre-inflammatory mesenchymal (PRIME) cells, and synovial macrophages in RA flare (Alivernini et al., 2020; Orange et al., 2020), it is also possible that exacerbation of disease is due to the loss of protective mechanisms in the setting of chronic joint inflammation. One area of interest receiving increased attention in RA is the synovial lymphatic system and its dysfunction, based on animal models and clinical pilots, where the loss of lymphatic drainage of inflamed joints is strongly implicated in the onset of synovitis and disease progression (Bouta et al., 2015, 2018; Rahimi et al., 2016a).

Previously, we demonstrated that arthritic progression in knee joints of tumor necrosis factor-transgenic (TNF-Tg) mice is paralleled by dramatic changes in the draining lymph nodes (Proulx et al., 2007b; Li et al., 2011; Chen et al., 2013). These longitudinal imaging studies combining contrast enhanced (CE) MRI of the synovium and popliteal lymph node (PLN) (Proulx et al., 2007b), with quantification of lymphatic drainage via near infrared (NIR) imaging of an injected dye (indocyanine green, ICG) (Zhou et al., 2010), demonstrated that prior to detectable synovial hyperplasia in the knee, the adjacent PLN expands. This PLN expansion is associated with increased lymphangiogenesis, elevated volume, $\mathrm{CD}_{11 b^{+}}$macrophage infiltration, and the accumulation of a unique subset of $\operatorname{IgD}^{+} / \mathrm{CD} 23^{+} / \mathrm{CD} 21^{h i} \underline{\mathrm{B}}$ cells in inflamed nodes (B-in) (Proulx et al., 2007a, 2008;
Zhang et al., 2007; Guo et al., 2009; Li et al., 2010; Zhou et al., 2010; Kuzin et al., 2016). This asymptomatic "expansion" phase is followed by a sudden "collapse" of the PLN, which is objectively defined by quantitative CE-MRI or power Doppler ultrasound (PD-US) imaging of the PLN (Proulx et al., 2007b; Bouta et al., 2013). This collapse, which occurs at variable time intervals in $\sim 80 \%$ of TNF-Tg mice, is associated with B-in translocation from the lymph node follicles to LYVE-1 ${ }^{+}$lymphatic vessels of the paracortical sinuses. Thereafter, lymphatic drainage declines significantly due to loss of intrinsic lymphatic contractions and passive flow (Proulx et al., 2007a; Li et al., 2010, 2011; Chen et al., 2013). It was also demonstrated that B cell depletion therapy (BCDT) with anti-CD20 antibodies ameliorated knee flare by "clearing" the collapsed lymph node sinuses, and restoring passive lymphatic flow despite the continued absence of detectable lymphatic contractions (Chen et al., 2013).

In addition, we have previously reported that nitric oxide signaling is associated with the loss of the lymphatic pulse in TNF-Tg mice. Pharmacologic inhibition of inducible nitric oxide synthase (iNOS) using $\mathrm{L}^{-\mathrm{N}^{6}}$-(1-iminoethyl)lysine 5-tetrazoleamide (L-NIL) in TNF-Tg mice has been shown to recover PLV contractions and ICG clearance via NIR imaging (Liang et al., 2016). We have also recently demonstrated that PLN expansion during arthritic progression is dependent on iNOS by longitudinally monitoring PLN volumes with PD-US in TNF-Tg vs. TNF-Tg $\mathrm{x}$ iNOS ${ }^{-/-}$mice (Bell et al., 2019a). Global ablation of iNOS preserved PLV contraction frequency assessed by in vivo NIR-ICG imaging, which was associated with significantly reduced synovitis in female TNF-Tg mice that typically exhibit an accelerated disease course (Bell et al., 2019a,b). Thus, the specific features associated with nitric oxide signaling and PLV contractility in TNF-Tg mice warrants further investigation.

Moreover, we have previously identified specific cell types in the local inflammatory environment of TNF-Tg PLVs that actively produce iNOS and may directly interfere with lymphatic contractility in vivo. In TNF-Tg mice, there is a significant increase in stagnant $\mathrm{CD}_{1} \mathrm{~b}^{+}$cells within the lumen of the PLVs (Li et al., 2013) that progressively adhere to the lymphatic endothelial cells (LECs) (Bouta et al., 2017). Moreover, a recent work also demonstrated that TNF-Tg LECs themselves produce significantly greater iNOS (eightfold higher mRNA) compared to wild-type (WT) controls (Liang et al., 2016). Through isolation of the PLVs ex vivo, we aimed to separate the specific extrinsic effects on contractility of the surrounding peri-lymphatic immune cells from the intrinsic defects within the LECs and lymphatic muscle cells (LMCs) of the PLV.

Additionally, lymphatic dysfunction in RA has also been demonstrated in clinical pilot studies. Manzo et al. (2011) used PD-US to show that joint draining lymph nodes in RA 
patients are subjected to subclinical intra-parenchymal changes and vascular flow modulation. Subsequently, we used CEMRI to show that RA patients with the smallest change in lymph node volume during anti-TNF therapy experienced the greatest pain relief in symptomatic knee joints with a remarkably linear inverse correlation (Rahimi et al., 2016b). Most recently, we utilized NIR-ICG imaging (Rahimi et al., 2016a) to show that lymphatic drainage in the hands of RA patients with active disease is decreased compared to healthy controls (Bell et al., 2020). Furthermore, this reduced lymphatic drainage was associated with a decrease in the total length of $\mathrm{ICG}^{+}$lymphatic vessels on the dorsal surface of the hands, which continued to contract at a similar rate as controls (Bell et al., 2020). Collectively, these findings support the hypothesis that there is an intrinsic defect in RA joint draining lymphatic vessels in advanced disease and/or flare, which warrants direct testing via ex vivo functional analyses. To this end, we utilized our ex vivo approaches to assess the contractile function of PLVs from WT and TNF-Tg mice under conditions of controlled hydrostatic and oncotic pressures in the absence of flow (Scallan et al., 2016; Zawieja et al., 2017; Castorena-Gonzalez et al., 2018). Moreover, real-time diameter tracking of ex vivo PLV pump function in this system allows for quantification of end diastolic diameter (EDD), tone, amplitude (AMP), ejection fraction (EF), contraction frequency (FREQ), and fractional pump flow (FPF). We also assessed alterations in these outcome measures in WT and TNF-Tg PLVs following non-specific NOS inhibition using $\mathrm{N} \omega$-nitro-L-arginine methyl ester (L-NAME). Here we report initial findings with this methodology, which demonstrate progressive intrinsic defects in PLVs from TNF-Tg mice with inflammatory-erosive arthritis, some of which are dependent on NOS activity.

\section{MATERIALS AND METHODS}

\section{Animals and Treatment}

All animal research was conducted on IACUC approved protocols. TNF-Tg mice (3,647 line) (Keffer et al., 1991) were originally acquired from Dr. G. Kollias (kollias@fleming.gr) and the Fleming Institute in Athens, Greece, and are maintained as heterozygotes in a C57BL/6 background with WT littermates used as controls. Only male mice were used in this study as female mice reach early mortality due to cardiopulmonary failure (Bell et al., 2019b). Mice in the Expanded cohort were 4-5months-old, while those in the Collapsed group were 9-11months-old. In the TNF-Tg mice, PLN Expansion occurs with limited joint disease, while PLN Collapse is concomitant with onset of severe synovitis and erosions in associated joints ( $\mathrm{Li}$ et al., 2011). For all imaging, mice were anesthetized with 1.5$2 \%$ isoflurane. For tissue harvesting, mice were anesthetized with pentobarbital sodium and immediately following this procedure, euthanized by overdose.

Primers for genotyping of TNF-Tg mice:

Forward: 5'-TAC-CCC-CTC-CTT-CAG-ACA-CC-3' Reverse: 5'-GCC-CTT-CAT-AAT-ATC-CCC-CA-3'

\section{Power Doppler Ultrasound}

The popliteal lymph nodes (PLNs) of TNF-Tg mice were phenotyped as "Expanding" or "Collapsed" using PD-US as previously described (Bouta et al., 2013). PD-US was also performed on the knee joints to confirm disease severity as previously described (Bouta et al., 2014). Each joint was imaged with a high-resolution small-animal ultrasound system (VisualSonics 770, Toronto, Ontario, Canada) using a 704b scanhead.

\section{Near Infrared Indocyanine Green Imaging}

Mice were placed on a heated surface (Indus Instruments, Webster, TX, United States), hair was removed with a depilatory cream and the mouse footpad was injected with $10 \mu \mathrm{l}$ of 0.1\% ICG (Akorn, Lake Forest, Illinois, United States) as previously described (Li et al., 2013). The imaging system was composed of a lens (Zoom 7000, Navitar, Rochester, NY, United States), ICG filter set (Semrock, Lake Forest, IL, United States) and camera (Prosilica GT1380, Allied Vision Technologies, Exton, PA, United States). ICG was excited with a tungsten halogen bulb (IT 9596ER, Illumination Technologies, Inc., Syracuse, NY, United States) through a ring illuminator (Schott, Elmsford, NY, United States). Imaging settings and recordings were accomplished through a custom built LabVIEW program (National Instruments, Austin, TX, United States). Real time NIR imaging was performed for $60 \mathrm{~min}$ after ICG injection into the footpad to quantify the lymphatic contraction rate, and mice were imaged $24 \mathrm{~h}$ later to quantify lymphatic clearance as previously described (Li et al., 2013).

\section{Vessel Isolation Procedure}

PLVs $(n=5)$ were harvested from WT and TNF-Tg mice as previously described (Scallan and Davis, 2013; CastorenaGonzalez et al., 2018). Briefly, the mice were anesthetized with pentobarbital sodium (Nembutal; $60 \mathrm{mg} \mathrm{kg}^{-1}$, i.p.) and placed in the prone position on a heating pad. An anterolateral incision $(\sim 1 \mathrm{~cm})$ was made in the skin beginning at the ankle of one leg to expose the two PLVs adjacent to the superficial saphenous vein. The superficial saphenous vein serves as a landmark to locate the two PLVs that run parallel to the vein. After the connective tissue on either side of the vein was cleared away, the more superficial of two PLVs was then separated from the vein and placed in Krebs buffer containing albumin. Afterward, the animal was euthanized by an overdose of pentobarbital sodium (200 $\mathrm{mg} \mathrm{kg}^{-1}$, i.p.). PLVs $(\sim 40-80$ $\mu \mathrm{m}$ i.d.; 1-2 $\mathrm{mm}$ long) were pinned in a Sylgard dish and cleaned of connective and adipose tissue before transfer to a $3 \mathrm{ml}$ chamber where the vessel was cannulated, pressurized, and trimmed of any remaining connective tissue prior to beginning the experimental protocol.

\section{Solutions and Chemicals}

Krebs buffer contained (in $\mathrm{mM}$ ): $\mathrm{NaCl}, 146.9 ; \mathrm{KCl}, 4.7$; $\mathrm{CaCl}_{2} \cdot 2 \mathrm{H}_{2} \mathrm{O}, 2 ; \mathrm{MgSO}_{4}, 1.2 ; \mathrm{NaH}_{2} \mathrm{PO}_{4} \cdot \mathrm{H}_{2} \mathrm{O}, 1.2 ; \mathrm{NaHCO}_{3}, 3$; sodium-Hepes, 1.5; d-glucose, $5\left(\mathrm{pH} 7.4\right.$ at $\left.37^{\circ} \mathrm{C}\right)$. An identical buffer was prepared with the addition of $0.5 \%$ BSA. During 
cannulation, the luminal and abluminal solutions contained Krebs with BSA, but during the experiment the abluminal solution was constantly exchanged with fresh Krebs lacking BSA. For the L-NAME experiments, L-NAME was added to the Krebs buffer lacking BSA at a concentration $\left(1 \times 10^{-4}\right)$, which is sufficient to maximally inhibit eNOS activity (Bohlen et al., 2009; Nagai et al., 2011; Scallan and Davis, 2013). At the end of every experiment, a $\mathrm{Ca}^{2+}$-free physiological saline solution was used to obtain the passive diameter (Davis et al., 2011). All chemicals were obtained from Sigma (St. Louis, MO, United States), with the exception of BSA (US Biochemicals; Cleveland, $\mathrm{OH}$, United States), $\mathrm{MgSO}_{4}$ (Thermo Fisher Scientific; Pittsburgh, PA, United States) and sodium-Hepes (Thermo Fisher Scientific). Table 1 provides details of all key reagents.

\section{Pressure Control and Data Acquisition}

Vessels were cut to a length that contained only a single valve. To prevent continuous, but not pulsatile, flow through the vessel during the experiment, input and output pressures were kept equal (Gashev et al., 2002; Bohlen et al., 2009). PLV segments were tied onto two glass micropipettes (40 $\mu \mathrm{m}$ o.d.) mounted on a Burg-style V-track system (Duling et al., 1981). Polyethylene tubing (PE-190) attached to each micropipette was later connected to a valve that allowed pressure control to be switched between a manual reservoir and servo-controlled pumps (Davis et al., 2011). After the isolated vessel chamber was positioned on an inverted microscope, a suffusion line connected to a peristaltic pump maintained a constant superfusion of Krebs buffer at a rate of $0.4 \mathrm{ml}$ $\min ^{-1}$; a second line attached to the peristaltic pump in reverse orientation was used to remove excess buffer at the same rate. Input and output pressures were set briefly to the highest pressure used in this study $\left(10 \mathrm{cmH}_{2} \mathrm{O}\right)$ to facilitate the removal of axial slack, which minimized bowing of the vessel at high pressures that otherwise interfered with diameter tracking. Afterward, both pressures were lowered to $3 \mathrm{cmH}_{2} \mathrm{O}$ to allow the vessel to warm up to $37^{\circ} \mathrm{C}$ over the course of $1 \mathrm{~h}$ and begin contracting. Thus, contractile function was assessed beginning approximately $2 \mathrm{~h}$ after the vessels were removed from the animal.
The input and output pressure transducer signals were recorded on a computer, and displayed a video image of the vessel using a firewire camera (model A641FM Basler; Ahrensburg, Germany) at $30 \mathrm{~Hz}$. A custom-written LabView program (National Instruments; Austin, TX, United States) measured the inner diameter (i.d.) of the vessel on the video image and recorded it as a function of time (Davis et al., 2011). Each PLV was equilibrated for $30-60 \mathrm{~min}$ at $2-3 \mathrm{cmH}_{2} \mathrm{O}$ and $37^{\circ} \mathrm{C}$ until a stable pattern of spontaneous contractions developed. Spontaneous contractions were recorded at each pressure for 2-6 $\mathrm{min}$, a time sufficient to obtain at least three contractions at the lowest pressure of $0.5 \mathrm{cmH}_{2} \mathrm{O}$. After the last pressure step to $10 \mathrm{cmH}_{2} \mathrm{O}$, pressures were lowered to $3 \mathrm{cmH}_{2} \mathrm{O}$ and the contraction pattern was allowed approximately $20 \mathrm{~min}$ to stabilize. All diameters reported here are inner diameters, and EDD, tone, amplitude (AMP), ejection fraction (EF), contraction frequency (FREQ), and fractional pump flow (FPF) were quantified as previously described (Scallan and Davis, 2013).

\section{Statistical Analysis}

Data obtained from the pressure step protocol were plotted as a function of pressure $\left(\mathrm{cmH}_{2} \mathrm{O}\right)$. Raw pressure/diameter traces were plotted against time using Igor Pro (Wavemetrics, Lake Oswego, OR, United States). To compare responses obtained within the same vessel, a repeated-measures two-way ANOVA was used in conjunction with Bonferroni's post hoc test. All data were tabulated using Excel, and statistical tests were performed using Prism 5 (Graphpad Software Inc., CA, United States), with significance for all tests set at $P<0.05$ and reported as means $\pm($ SEM $)$.

\section{RESULTS}

\section{Ex vivo Popliteal Lymphatic Vessels From TNF-Transgenic Mice Exhibit Distinct Functional Deficits}

Gross visualization of WT (Movie 1), Expanded (Movie 2), and Collapsed (Movie 3) PLV contractions revealed that the TNF-Tg PLVs appeared to be larger in diameter, and had

TABLE 1 | Key experimental reagents.

\begin{tabular}{|c|c|c|c|c|}
\hline & Reagents & Vendor & Catalog number & Concentration \\
\hline 2 & $\mathrm{NaCl}$ & Sigma & S9888 & $146.9 \mathrm{mM}$ \\
\hline 4 & $\mathrm{CaCl}_{2} \cdot 2 \mathrm{H}_{2} \mathrm{O}$ & Sigma & 223506 & $2 \mathrm{mM}$ \\
\hline 5 & $\mathrm{MgSO}_{4}$ & Thermo Fisher Scientific & M65 & $1.2 \mathrm{mM}$ \\
\hline 6 & $\mathrm{NaH}_{2} \mathrm{PO} 4 \bullet \mathrm{H}_{2} \mathrm{O}$ & Sigma & S9638 & $1.2 \mathrm{mM}$ \\
\hline 8 & Sodium-Hepes & Thermo Fisher Scientific & BP410 & $1.5 \mathrm{mM}$ \\
\hline 9 & d-Glucose & Sigma & G8270 & $5 \mathrm{mM}$ \\
\hline 10 & BSA & US Biochemicals & CAS: 9048-46-8 & $0.5 \%$ \\
\hline 11 & Krebs Buffer $=2-9$ & NA & NA & NA \\
\hline
\end{tabular}

The table lists the reagent, vendor, specific catalog number or unique chemical identifier, and concentration used for this study. 

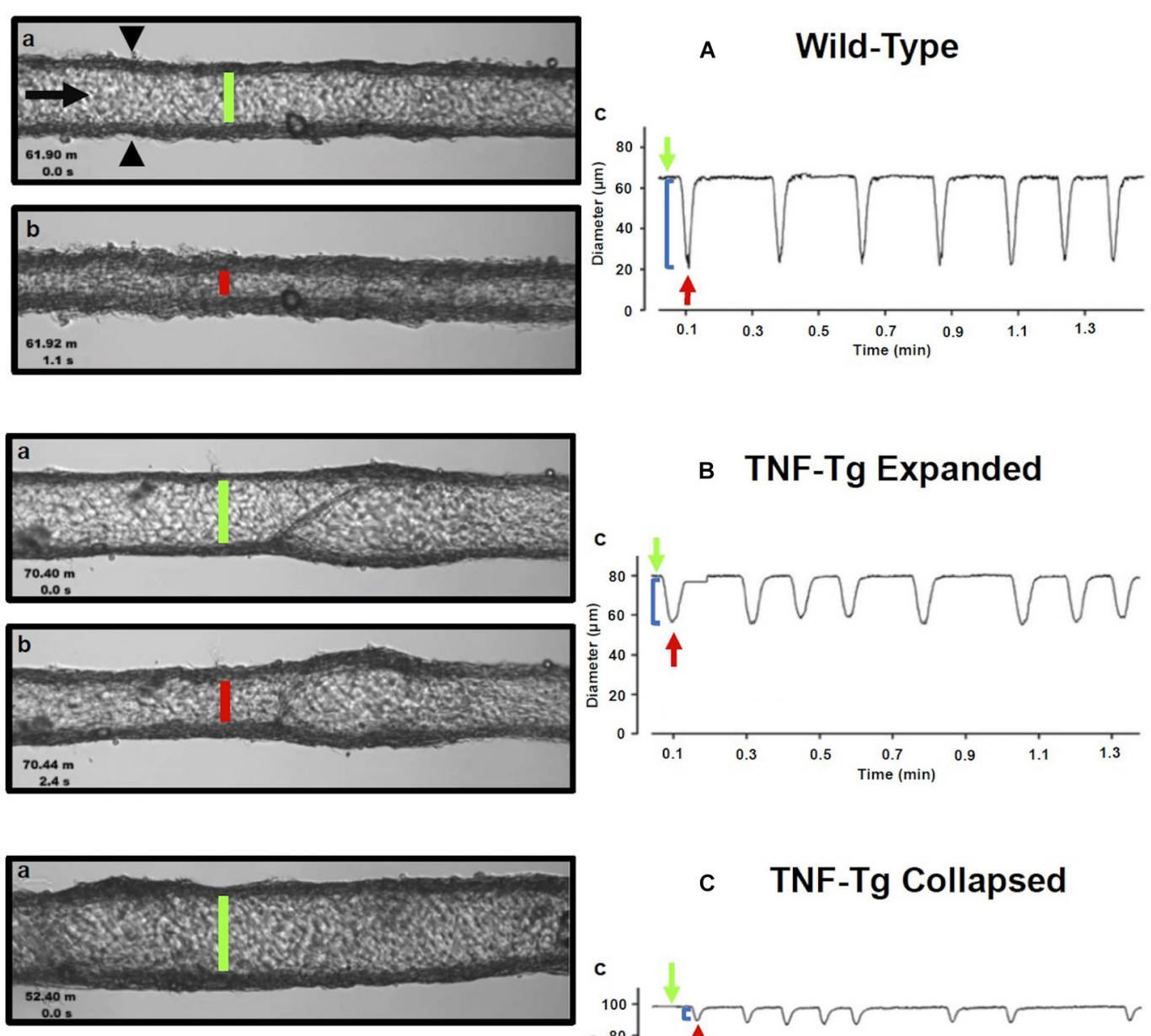

c TNF-Tg Collapsed
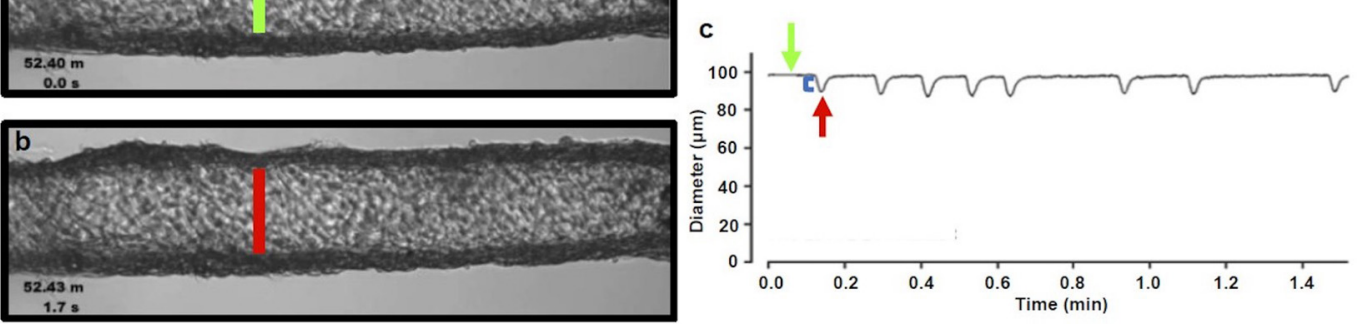

FIGURE 1 | Isolated popliteal lymphatic vessels from TNF-Tg mice exhibit a progressive reduction in contractility. Popliteal lymphatic vessels (PLVs) were harvested from wild-type (age-matched littermates) (A) and TNF-Tg mice with expanded (4-5-months-old) (B) and collapsed (9-11 months-old) (C) popliteal lymph nodes ( $n=5$ mice per group). The PLVs were then cannulated with constant fluid flow into the lumen of the vessel (black arrow) to measure spontaneous PLV contractions by assessing changes in diameter between the vessel walls (black arrowheads) (Aa). In each condition, representative PLVs are shown in diastole (A-Ca) and systole (A-Cb) with quantified changes in diameter representing contractions (local minima) over time under constant pressure $\left(3 \mathbf{c m H} \mathrm{H}_{2} \mathrm{O}\right)(\mathbf{A}-\mathbf{C c})$. Note the increase in end diastolic diameter (EDD; green lines) and end systolic diameter (ESD; red lines) in TNF-Tg PLVs (expanded < collapsed) compared to wild-type PLVs (A-Ca,b). The change in EDD (green arrows) and ESD (red arrows) represents a reduction in contraction amplitude (blue brackets) in TNF-Tg PLVs (expanded > collapsed) relative to wild-type control PLVs (A-Cc).

much weaker/ineffective contractions compared to WT, but no differences in contraction frequency were observed (Figure 1). Although quantification of the EDD failed to detect significant differences between the groups, it did reveal a trend of increased EDD of Collapsed PLV at all pressures tested (Figure 2A). We also observed a consistent trend of decreased tone in both Expanding and Collapsed PLV vs. WT at all pressures, which did not reach statistical significance (Figure 2B). The combination of increased EDD and decreased tone likely counteracted each other leading to data that were not significant.
Formal quantification of the PLV contractions confirmed the apparent deficiencies in contractile amplitude (AMP) and ejection fraction (EF) observed in the videos. The contraction AMP and EF of both Expanding and Collapsed PLVs were significantly decreased vs. WT at pressures of $0.5-5.0 \mathrm{cmH}_{2} \mathrm{O}$ $(P<0.05)$ (Figures 2C,D). Also consistent with the videos, we did not observe differences in PLV contraction frequency at any pressure (Figure 2E). However, despite normal contraction frequency, Expanding and Collapsed PLVs showed significantly decreased FPF at pressures of 2.0-5.0 $\mathrm{cmH}_{2} \mathrm{O}$ vs. WT 
A

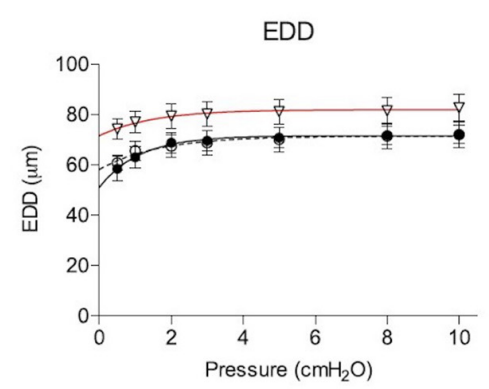

C

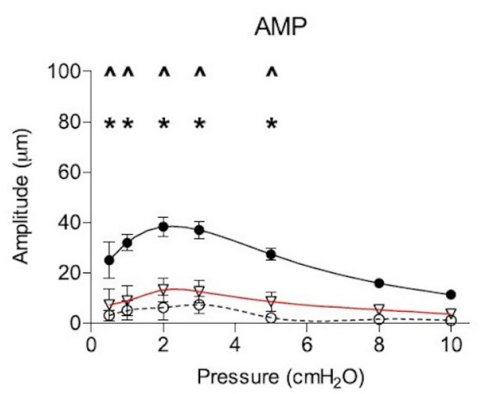

E

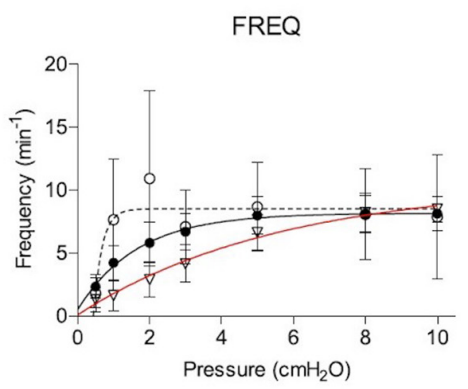

B

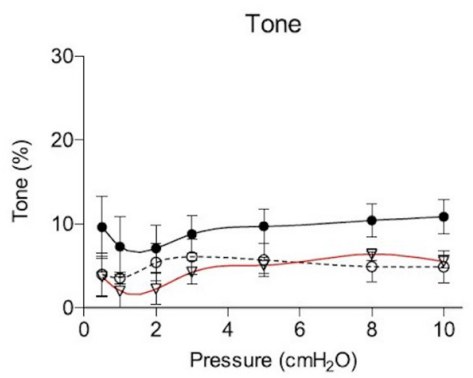

D

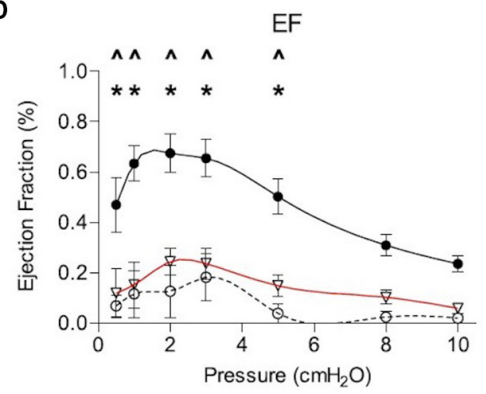

$\mathbf{F}$

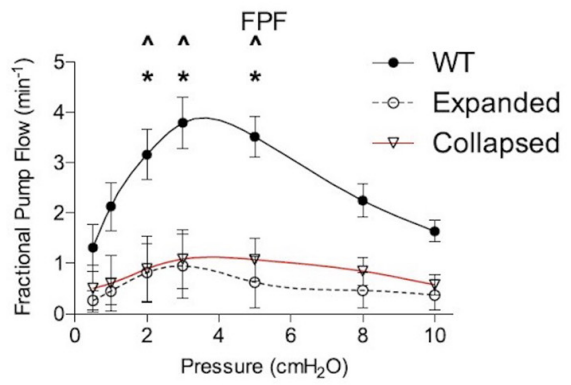

FIGURE 2 | Expanding and Collapsed TNF-Tg mice show reduced amplitude (AMP), ejection fraction (EF), and fractional pump flow (FPF) compared to WT. Lymphatic contractile parameters are plotted against pressure on the $x$-axis, and include end diastolic diameter (EDD; A), tone (B), contraction amplitude (AMP; C), ejection fraction (EF; D), contraction frequency (FREQ; E) and FPF (F). All data are means ( \pm SEM). When error bars appear missing, they are actually contained within the data points. ${ }^{*} P<0.05$ Expanded vs. WT, ${ }^{\wedge} P<0.05$ Collapsed vs. WT.

(Figure 2F), demonstrating the severity of the TNF-induced defect in PLV contraction strength under these conditions.

\section{Functional Defects in TNF-Transgenic Popliteal Lymphatic Vessels Are Mediated by Nitric Oxide-Dependent Mechanisms}

To better understand the functional deficits noted in the TNFTg PLVs compared to WT controls, we assessed the role of nitric oxide in mediating the reduced contractility by treating the PLVs with and without L-NAME. In WT and Expanding PLVs, L-NAME administration did not produce any notable changes in tone, while Collapsed PLVs exhibited significantly increased tone at all pressures (except $2.0 \mathrm{cmH}_{2} \mathrm{O}$ ) (Figures 3A-C). Similarly, contraction frequency remained unchanged in WT (except 0.5 $\mathrm{cmH}_{2} \mathrm{O}$ ) and Expanding PLVs, whereas Collapsed PLVs showed significantly increased contraction frequency at all pressures after treatment with L-NAME (Figures 3D-F). Following L-NAME treatment, WT and Expanding PLVs demonstrated a significant increase in FPF at pressures $\leq 3.0 \mathrm{cmH}_{2} \mathrm{O}$ (Figures $3 \mathbf{G}, \mathbf{H}$ ). However, Collapsed PLVs demonstrated significant increases in FPF at all pressures following L-NAME administration (Figure 3I). These results indicate a substantial role for nitric oxide signaling in the reduction of PLV contractility during the Collapsed phase of TNF-mediated inflammatory arthritis.

Nitric oxide synthase inhibition improved many of the functional defects in TNF-Tg PLVs when compared to untreated WT levels (Figure 4). As expected from the untreated PLVs (Figure 2), EDD, and thus \% tone, remained unchanged between the groups after L-NAME administration (Figures 4A,B). L-NAME also reversed the contractile defects in AMP noted in Collapsed PLVs at pressures of 0.5-5.0 $\mathrm{cmH}_{2} \mathrm{O}$ (Figure 2C vs. Figure 4C). However, L-NAME was unable to significantly 

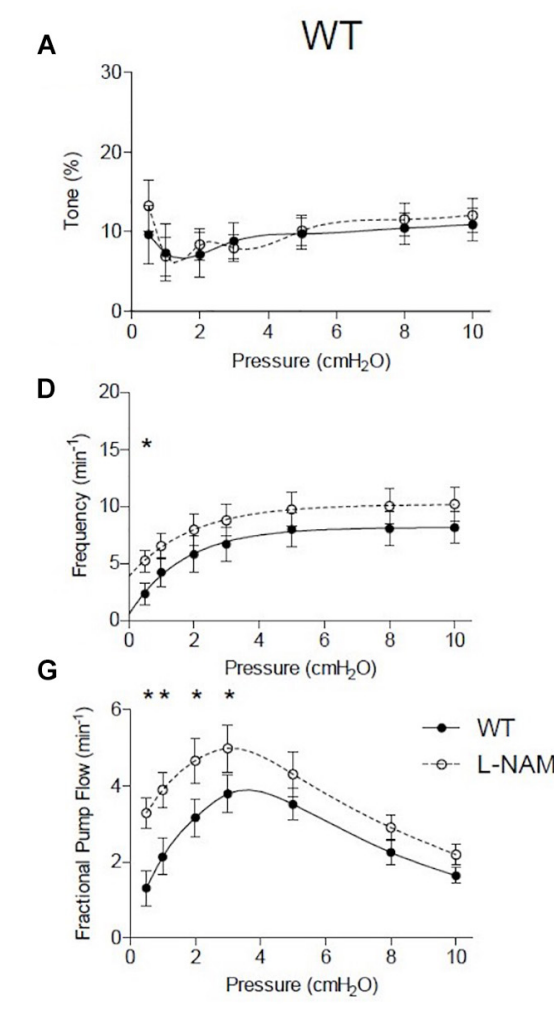

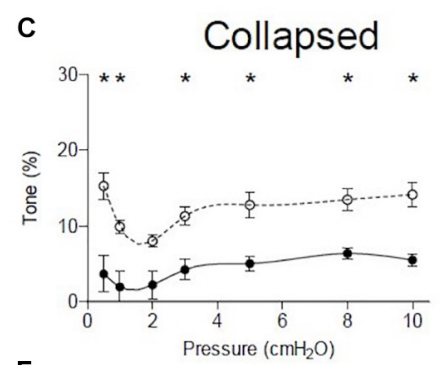

E

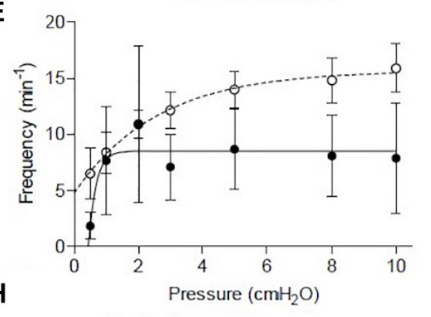

F

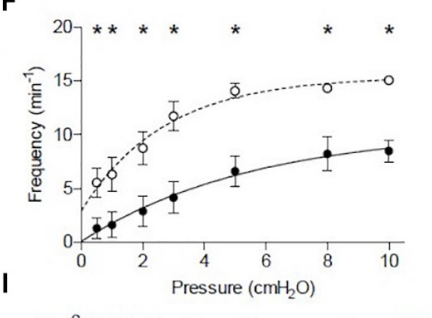

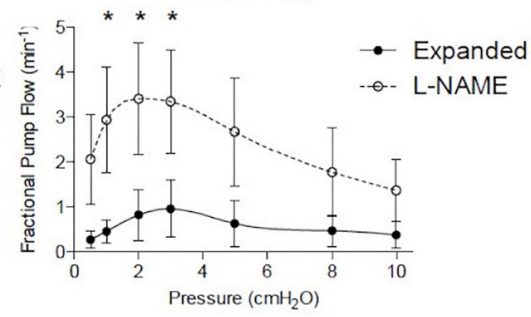

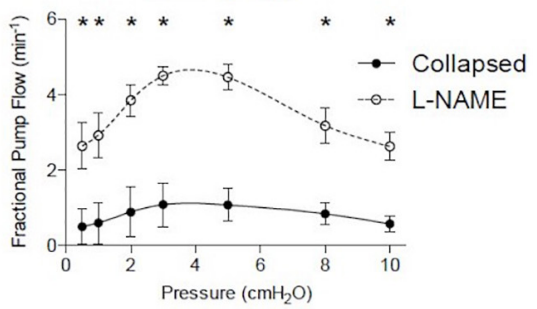

FIGURE 3 | Collapsed lymphatic vessels display distinct nitric oxide-dependent functional defects ex vivo. The lymphatic vessels described in Figure $\mathbf{1}$ were treated with and without L- NAME $\left(10^{-4} \mathrm{M}\right)$, and their tone, contraction frequency and fractional pump flow vs. pressure were determined ex vivo. All data are means ( \pm SEM). When error bars appear missing, they are actually contained within the data points ( $P<0.05$ vs. L-NAME at the same pressure). Note that L-NAME has very limited effects on vessels afferent to WT and Expanding PLN, but restores tone, frequency, and fractional pump flow in lymphatic vessels afferent to Collapsed PLN at all pressures tested.

correct the contractile defect in AMP in Expanding PLVs at the same pressures (Figure 4C), indicating the presence of a nitric oxide-independent mechanism that may also contribute to contractile defects prior to the Collapsed stage. Both EF and FPF, which showed significant reductions in both Expanding and Collapsed TNF-Tg PLVs (Figures 2D,F), demonstrated dramatic recovery of these outcome measures to WT levels when treated with L-NAME (Figures 4D,F) indicating a role for nitric oxide in these defects. Finally, L-NAME treatment dramatically improved the contraction frequency of both Expanding and Collapsed PLVs beyond WT levels, but this was significant only at the highest two pressures $\left(8-10 \mathrm{cmH}_{2} \mathrm{O}\right)$.

\section{DISCUSSION}

In this study, we utilized ex vivo methodologies to directly assess functional differences between PLVs from TNF-Tg mice with Expanding and Collapsed PLNs, vs. PLVs from their WT littermates. The results indicated significant deficiencies in TNF-Tg PLVs, which formally demonstrates the intrinsic defects in joint draining lymphatic function predicted by in vivo imaging and histological analyses. Thus, the lack of ICG clearance following foot pad injection of mice with inflammatory arthritis (Bouta et al., 2018), and in the hands of patients with symptomatic RA (Bell et al., 2020), is likely due to the loss of lymphatic vessel contractile function that we observed as decreases in AMP, EF, and FPF. In addition, non-selective inhibition of NOS using L-NAME demonstrated recovery of AMP, EF, and FPF in the Collapsed TNF-Tg PLVs, but the vessels continued to exhibit decreased AMP during the Expanding phase. The specific contributions of endothelial nitric oxide (eNOS) and iNOS on these outcome measures through administration of L-NIL would be interesting to investigate in future studies given the proposed isolated role of iNOS on the deficits of TNF-Tg lymphatic contractility in vivo (Bell et al., 2019a). Of note, the retained deficiency in AMP during the Expanded phase following NOS inhibition ex vivo (Figure 4C), and the incomplete resolution of lymphatic function following global iNOS ablation in vivo (Bell et al., 2019a), suggests additional mechanisms are likely associated with the deficiencies in PLV contractility during the progression of inflammatory arthritis (i.e., structural damage and lymphatic muscle cell apoptosis; Bouta et al., 2017), which is an active area of investigation. In addition, future studies to evaluate the return of contractile deficiencies after washout of L-NAME, or the effects of anti-TNF therapy on TNF-Tg PLVs ex vivo ought to be conducted to clarify the cellular mechanisms of the contractile dysfunction. 


\section{A}

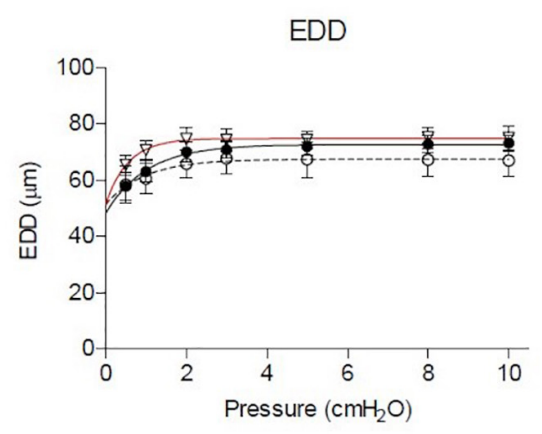

C

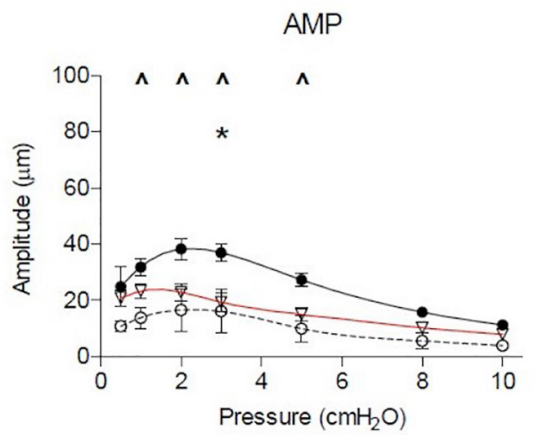

E

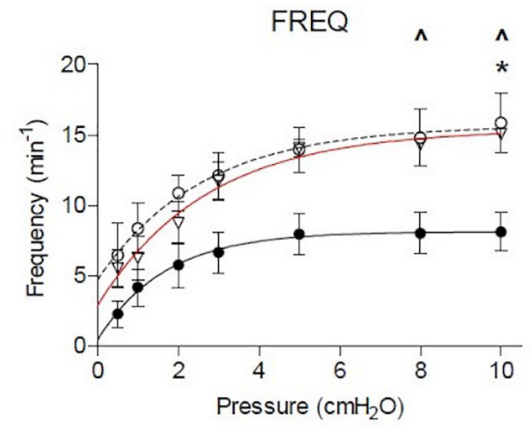

B

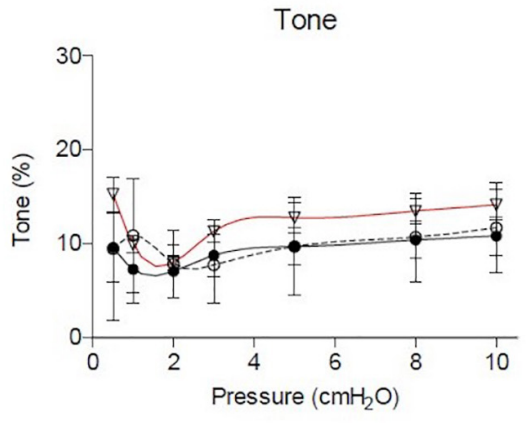

D

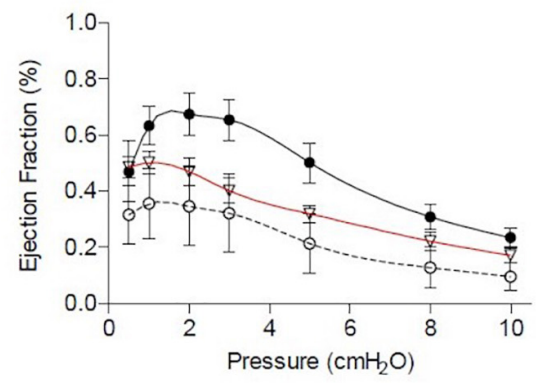

$\mathbf{F}$

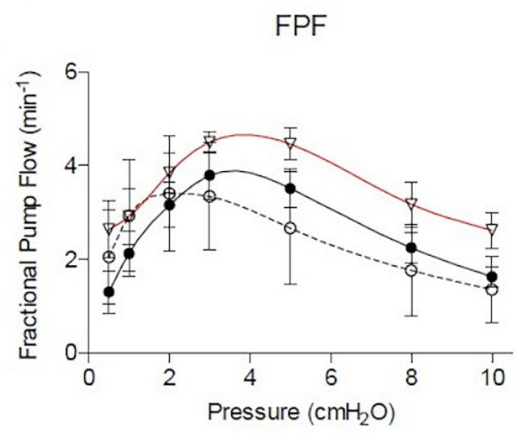

FIGURE 4 | Nitric oxide synthase inhibition corrects TNF-Tg lymphatic vessel defects in contractile function. The lymphatic vessels described in Figure 1 were treated with L-NAME $\left(10^{-4} \mathrm{M}\right)$ and assessed for EDD, \% tone, AMP, EF, FREQ, and FPF at the indicated pressures. All data are means ( \pm SEM). When error bars appear missing, they are contained within the data points ( ${ }^{\star} P<0.05$ Expanded + L-NAME vs. WT; $\wedge P<0.05$ Collapsed + L-NAME vs. WT).

Previously, we have focused on the synovial lymphatic system during arthritic progression and flare (Bouta et al., 2018), and the effects of interventions that specifically target lymphatic contractions, whose physiologic importance in supporting lymphatic drainage and subsequent onset of lymphedema have been established in preclinical and clinical studies (Mallick and Bodenham, 2003; Zawieja, 2009; Bouta et al., 2018; Bell et al., 2020). We have also performed transmission electron microscopy studies on WT, Expanding and Collapsed PLVs
(Bouta et al., 2017). These studies confirmed that large, activated macrophages attach to damaged endothelial cells in Expanding PLVs of TNF-Tg mice with early arthritis, and that lymphatic muscle cells undergo apoptosis in Collapsed PLVs of TNF-Tg mice with advanced arthritis. Remarkably, this TNF-mediated damage was reversible with anti-TNF therapy that ameliorated the erosive inflammatory arthritis, in part via restoration of PLV contractions and potential enhancement of inflammatory cell egress (Bouta et al., 2017). However, while these studies 


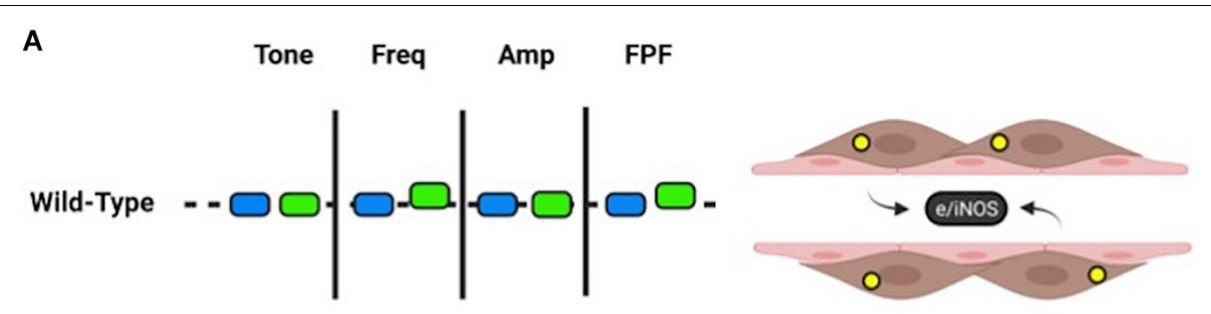

B

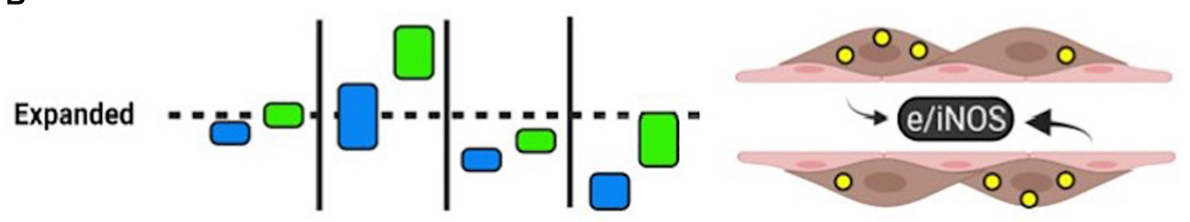

C
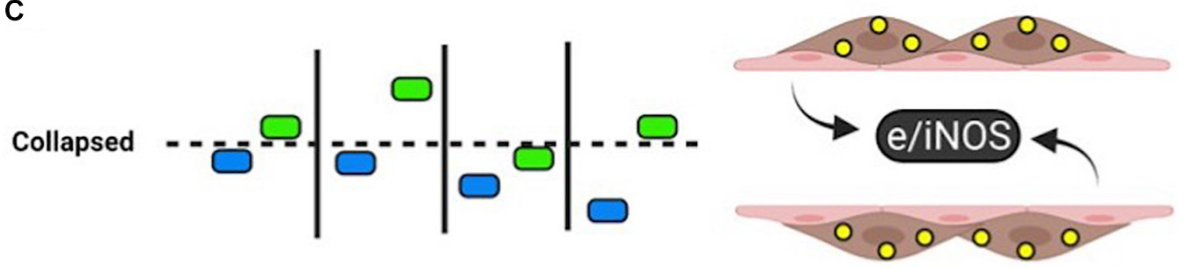

FIGURE 5 | Summary of WT and TNF-Tg contractility with and without L-NAME. Wild-type PLVs exhibit homeostatic NOS (produced by pink LECs ex vivo) and NO levels (yellow circles within brown LMCs). L-NAME (green rectangles) leads to slight increases in FREQ and FPF from baseline (blue rectangles). Approximate changes are demonstrated by vertical movement up (increased) or down (decreased) of the rectangles (A). Relative to wild-type baselines (dashed line), expanded TNF-Tg PLVs have similar tone, increased variability of FREQ (vertical length of rectangle), reduced AMP, and reduced FPF. L-NAME shows an increased trend in all measures of contractility, while FREQ and FPF exhibit notable variability between expanded TNF-Tg PLVs (B). PLVs from collapsed TNF-Tg mice showed comparable effects to expanded TNF-Tg PLVs, but with uniform measures of FREQ and FPF. L-NAME dramatically increased tone, FREQ, and FPF to wild-type levels or above (C). For all groups, L-NAME treatment stabilized FPF to baseline levels primarily associated with increased FREQ of TNF-Tg PLVs. Given the variability of FREQ in expanded TNF-Tg PLVs, as a group this stage of disease may be less dependent on NOS inhibition to recover FPF compared to collapsed TNF-Tg PLVs with more consistent FREQ responses. Created with BioRender.com.

strongly implicate intrinsic PLV defects in the progression and flare of joint disease in TNF-Tg mice, in vivo studies cannot isolate PLV function independent of the upstream inflammation in the arthritic joint, and potential immune reactions in the efferent PLN.

In fact, the temporal dynamics between PLN Collapse and reduced PLV contractility continue to be an essential focus of research in the TNF-Tg mice to determine which phenomenon is the inciting event. While current studies are actively investigating the mechanisms of PLN collapse, this work provides valuable insight to suggest that reduced PLV contractility precedes PLN collapse. We demonstrated for the first time that isolated PLVs from TNF-Tg mice with both Expanding and Collapsed PLNs exhibit persistent contractile deficiencies. Thus, we believe this study provides evidence that the consistent decline in lymphatic contractility in TNF-Tg mice initiates and eventually culminates in stagnation of lymphatic flow leading to PLN Collapse.

Proper contractility of a lymphatic vessel requires a dynamic interplay between LMCs and LECs. As measured by the contractility of the TNF-Tg vessels ex vivo, there is a significant decrease in FPF driven predominately by reduced AMP and
EF without a compensatory increase in FREQ to maintain FPF. Remarkably, L-NAME treatment was able to dramatically increase the FREQ of TNF-Tg PLVs, while overall AMP remained decreased (Figure 5). As TNF-Tg LECs are known to produce significantly greater iNOS than those of WT PLVs, our data suggests that the L-NAME treatment is predominately affecting lymphatic contractility through LECs. However, the persistence of reduced AMP indicates that the lymphatic deficiencies do not function solely through NOS production by LECs. A recently published study demonstrated that the treatment of primary LMC cultures with TNF was associated with reduced mRNA expression of key contractile and cytoskeletal proteins (Liang et al., 2021). Similarly, chronic TNF exposure in TNF-Tg mice may reduce expression of essential LMC contractile proteins associated with the noted persistence of certain deficiencies in contractility ex vivo, and this is an active area of investigation.

We also observed some apparent inconsistencies with the in vivo data, most notably the absence of functional differences between Expanding and Collapsed PLVs, and the normal contraction frequency of Collapsed PLVs ex vivo. To interpret these discrepancies correctly, it is critical to note that Collapsed PLVs in vivo are filled with static monocytes and macrophages 
(Li et al., 2013; Bouta et al., 2017), which are removed during the cannulation and preparations prior to ex vivo analysis. Thus, it may be that these resident immune cells, known to express high levels of iNOS and inflammatory cytokines (Bell et al., 2019a), may be responsible for additional or more dramatic PLV deficits that were not detected in our ex vivo study. In addition, contraction frequency measured in vivo by NIRICG imaging requires lymphatic contractions with sufficient force to generate bolus flow of ICG through the vessel. While the pacemaker activity seems to remain intact in Collapsed PLVs based on the unchanged contraction frequency ex vivo (Figures 1C, 2E), other factors, such as a proposed lymphatic valvular insufficiency in Collapsed PLVs (Li et al., 2013; Bouta et al., 2015), could not be assessed in our ex vivo studies. Another potential confounding caveat is that the additional environmental or functional factors of the PLVs in vivo, coupled with the reduced AMP, EF, and FPF noted ex vivo, may not produce sufficient fluid flow to be visualized by in vivo NIR-ICG imaging. Thus, while the measures of in vivo and ex vivo contraction frequency may seem inconsistent for Collapsed PLVs, the in vivo measures may represent a loss of "effective" contractions associated with successful anterograde lymphatic drainage.

In summary, we show for the first time that PLVs from TNF$\mathrm{Tg}$ mice with inflammatory arthritis have intrinsic functional defects that result in significant contractile dysfunction. As these defects have been associated with arthritic progression and flare in animal models of RA and patients with symptomatic disease, these data provide direct evidence of isolated lymphatic vessel dysfunction, and further validate lymphatics as a therapeutic target. Thus, drugs and stem cell therapies specifically designed to improve lymphatic vessel repair and the return of homeostatic lymphatic contractions are potential treatments for RA that warrant future investigation.

\section{REFERENCES}

Alivernini, S., MacDonald, L., Elmesmari, A., Finlay, S., Tolusso, B., Gigante, M. R., et al. (2020). Distinct synovial tissue macrophage subsets regulate inflammation and remission in rheumatoid arthritis. Nat. Med. 26, 1295-1306. doi: 10.1038/ s41591-020-0939-8

Bell, R. D., Rahimi, H., Kenney, H. M., Lieberman, A. A., Wood, R. W., Schwarz, E. M., et al. (2020). Altered lymphatic vessel anatomy and markedly diminished lymph clearance in the rheumatoid hand with active arthritis. Arthritis Rheumatol. 72, 1447-1455. doi: 10.1002/art.41311

Bell, R. D., Slattery, P. N., Wu, E. K., Xing, L., Ritchlin, C. T., and Schwarz, E. M. (2019a). iNOS dependent and independent phases of lymph node expansion in mice with TNF-induced inflammatory-erosive arthritis. Arthritis Res. Ther. 21:240. doi: 10.1186/s13075-019-2039-Z

Bell, R. D., Wu, E. K., Rudmann, C. A., Forney, M., Kaiser, C. R. W., Wood, R. W., et al. (2019b). Selective sexual dimorphisms in musculoskeletal and cardiopulmonary pathologic manifestations and mortality incidence in the tumor necrosis factor-transgenic mouse model of rheumatoid arthritis. Arthritis Rheumatol. 71, 1512-1523. doi: 10.1002/art.40903

Bohlen, H. G., Wang, W., Gashev, A., Gasheva, O., and Zawieja, D. (2009). Phasic contractions of rat mesenteric lymphatics increase basal and phasic nitric oxide generation in vivo. Am. J. Physiol. Heart Circ. Physiol. 297, H1319-H1328. doi: 10.1152/ajpheart.00039.2009

Bouta, E. M., Banik, P. D., Wood, R. W., Rahimi, H., Ritchlin, C. T., Thiele, R. G., et al. (2014). Validation of power doppler versus contrast enhanced

\section{DATA AVAILABILITY STATEMENT}

The raw data supporting the conclusions of this article will be made available by the authors, without undue reservation.

\section{ETHICS STATEMENT}

The animal study was reviewed and approved by the Institutional Animal Care and Use Committee (IACUC) at the University of Rochester and University of Missouri.

\section{AUTHOR CONTRIBUTIONS}

JS, EB, HR, CR, MD, and ES contributed to conception and design of the study. ES wrote the first draft of the manuscript. JS, HK, and ES wrote sections of the manuscript. All authors contributed to manuscript revision, read, and approved the submitted version.

\section{FUNDING}

This work was supported by research grants from the National Institutes of Health (T32s GM007356 and AR076950; R01s HL-12256, HL-120867, HL-122578, HL-142905, AR069000, and AR056702; and P30 AR069655).

\section{SUPPLEMENTARY MATERIAL}

The Supplementary Material for this article can be found online at: https://www.frontiersin.org/articles/10.3389/fphys. 2021.745096/full\#supplementary-material

magnetic resonance imaging quantification of joint inflammation in murine inflammatory arthritis. J. Bone Mineral Res. : Off. J. Am. Soc. Bone Mineral Res. 30, 690-694. doi: 10.1002/jbmr.2392

Bouta, E. M., Bell, R. D., Rahimi, H., Xing, L., Wood, R. W., Bingham, C. O. 3rd, et al. (2018). Targeting lymphatic function as a novel therapeutic intervention for rheumatoid arthritis. Nat. Rev. Rheumatol. 14, 94-106. doi: 10.1038/ nrrheum.2017.205

Bouta, E. M., Ju, Y., Rahimi, H., de Mesy-Bentley, K. L., Wood, R. W., Xing, L., et al. (2013). Power doppler ultrasound phenotyping of expanding versus collapsed popliteal lymph nodes in murine inflammatory arthritis. PLoS One 8:e73766. doi: 10.1371/journal.pone.0073766

Bouta, E. M., Kuzin, I., de Mesy Bentley, K., Wood, R. W., Rahimi, H., Ji, R. C., et al. (2017). Treatment of tumor necrosis factor-transgenic mice with anti-tumor necrosis factor restores lymphatic contractions, repairs lymphatic vessels, and may increase monocyte/macrophage egress. Arthritis Rheumatol. 69, 1187-1193. doi: 10.1002/art.40047

Bouta, E. M., Li, J., Ju, Y., Brown, E. B., Ritchlin, C. T., Xing, L., et al. (2015). The role of the lymphatic system in inflammatory-erosive arthritis. Semin. Cell Dev. Biol. 38, 90-97. doi: 10.1016/j.semcdb.2015.01.001

Castorena-Gonzalez, J. A., Scallan, J. P., and Davis, M. J. (2018). Methods for assessing the contractile function of mouse lymphatic vessels ex vivo. Methods Mol. Biol. 1846, 229-248. doi: 10.1007/978-1-4939-8712-2_15

Chen, J., Cui, Y., Li, X., Miao, X., Wen, Z., Xue, Y., et al. (2013). Risk factors for deep infection after total knee arthroplasty: a meta-analysis. Arch. Orthop. Trauma Surg. 133, 675-687. doi: 10.1007/s00402-013-1723-8 
Davis, M. J., Rahbar, E., Gashev, A. A., Zawieja, D. C., and Moore, J. E. Jr. (2011). Determinants of valve gating in collecting lymphatic vessels from rat mesentery. Am. J. Physiol. Heart Circ. Physiol. 301, H48-H60. doi: 10.1152/ajpheart.00133. 2011

Duling, B. R., Gore, R. W., Dacey, R. G. Jr., and Damon, D. N. (1981). Methods for isolation, cannulation, and in vitro study of single microvessels. Am. J. Physiol. 241, H108-H116. doi: 10.1152/ajpheart.1981.241.1.H108

Firestein, G. S. (2003). Evolving concepts of rheumatoid arthritis. Nature 423, 356-361.

Firestein, G. S. (2014). The disease formerly known as rheumatoid arthritis. Arthritis Res. Ther. 16:114. doi: 10.1186/ar4593

Gashev, A. A., Davis, M. J., and Zawieja, D. C. (2002). Inhibition of the active lymph pump by flow in rat mesenteric lymphatics and thoracic duct. J. Physiol. 540(Pt 3), 1023-1037. doi: 10.1113/jphysiol.2001.016642

Guo, R., Zhou, Q., Proulx, S. T., Wood, R., Ji, R. C., Ritchlin, C. T., et al. (2009). Inhibition of lymphangiogenesis and lymphatic drainage via vascular endothelial growth factor receptor 3 blockade increases the severity of inflammation in a mouse model of chronic inflammatory arthritis. Arthritis Rheum. 60, 2666-2676. doi: 10.1002/art.24764

Keffer, J., Probert, L., Cazlaris, H., Georgopoulos, S., Kaslaris, E., Kioussis, D., et al. (1991). Transgenic mice expressing human tumour necrosis factor: a predictive genetic model of arthritis. EMBO J. 10, 4025-4031.

Kuzin, I. I., Kates, S. L., Ju, Y., Zhang, L., Rahimi, H., Wojciechowski, W., et al. (2016). Increased numbers of CD23(+) CD21(hi) Bin-like B cells in human reactive and rheumatoid arthritis lymph nodes. Eur. J. Immunol. 46, 1752-1757. doi: 10.1002/eji.201546266

Li, J., Ju, Y., Bouta, E., Xing, L., Wood, R., Kuzin, I., et al. (2013). Efficacy of B cell depletion therapy for murine joint arthritis flare is associated with increased lymphatic flow. Arthritis Rheumatol. 65:9.

Li, J., Kuzin, I., Moshkani, S., Proulx, S. T., Xing, L., Skrombolas, D., et al. (2010). Expanded CD23(+)/CD21(hi) B cells in inflamed lymph nodes are associated with the onset of inflammatory-erosive arthritis in TNF-transgenic mice and are targets of anti-CD20 therapy. J. Immunol. 184, 6142-6150. doi: 10.4049/ jimmunol.0903489

Li, J., Zhou, Q., Wood, R., Kuzin, I., Bottaro, A., Ritchlin, C. T., et al. (2011). CD23+/CD21hi B cell translocation and ipsilateral lymph node collapse is associated with asymmetric arthritic flare in TNF-Tg mice. Arthritis Res. Ther. 13:R138. doi: 10.1186/ar3452

Liang, Q., Ju, Y., Chen, Y., Wang, W., Li, J., Zhang, L., et al. (2016). Lymphatic endothelial cells efferent to inflamed joints produce iNOS and inhibit lymphatic vessel contraction and drainage in TNF-induced arthritis in mice. Arthritis Res. Ther. 18:62. doi: 10.1186/s13075-016-0963-8

Liang, Q., Zhang, L., Xu, H., Li, J., Chen, Y., Schwarz, E., et al. (2021). Lymphatic muscle cells contribute to dysfunction of the synovial lymphatic system in inflammatory arthritis in mice. Arthritis Res. Ther. 23:58. doi: 10.1186/s13075021-02438-6

Mallick, A., and Bodenham, A. R. (2003). Disorders of the lymph circulation: their relevance to anaesthesia and intensive care. Br. J. Anaesth. 91, 265-272.

Manzo, A., Caporali, R., Vitolo, B., Alessi, S., Benaglio, F., Todoerti, M., et al. (2011). Subclinical remodelling of draining lymph node structure in early and established rheumatoid arthritis assessed by power Doppler ultrasonography. Rheumatology (Oxford) 50, 1395-1400. doi: 10.1093/rheumatology/ker076

Nagai, T., Bridenbaugh, E. A., and Gashev, A. A. (2011). Aging-associated alterations in contractility of rat mesenteric lymphatic vessels. Microcirculation 18, 463-473. doi: 10.1111/j.1549-8719.2011.00107.x

Orange, D. E., Yao, V., Sawicka, K., Fak, J., Frank, M. O., Parveen, S., et al. (2020). RNA Identification of PRIME cells predicting rheumatoid arthritis flares. N. Engl. J. Med. 383, 218-228. doi: 10.1056/NEJMoa2004114

Pratt, A. G., and Isaacs, J. D. (2014). Seronegative rheumatoid arthritis: pathogenetic and therapeutic aspects. Best Pract. Res. Clin. Rheumatol. 28, 651-659. doi: 10.1016/j.berh.2014.10.016

Proulx, S. T., Kwok, E., You, Z., Beck, C. A., Shealy, D. J., Ritchlin, C. T., et al. (2007a). MRI and quantification of draining lymph node function in inflammatory arthritis. Ann. N. Y. Acad. Sci. 1117, 106-123.

Proulx, S. T., Kwok, E., You, Z., Papuga, M. O., Beck, C. A., Shealy, D. J., et al. (2007b). Longitudinal assessment of synovial, lymph node, and bone volumes in inflammatory arthritis in mice by in vivo magnetic resonance imaging and microfocal computed tomography. Arthritis Rheum. 56, 4024-4037.

Proulx, S. T., Kwok, E., You, Z., Papuga, M. O., Beck, C. A., Shealy, D. J., et al. (2008). Elucidating bone marrow edema and myelopoiesis in murine arthritis using contrast-enhanced magnetic resonance imaging. Arthritis Rheum. 58, 2019-2029. doi: 10.1002/art.23546

Rahimi, H., Bell, R., Bouta, E. M., Wood, R. W., Xing, L., Ritchlin, C. T., et al. (2016a). Lymphatic imaging to assess rheumatoid flare: mechanistic insights and biomarker potential. Arthritis Res. Ther. 18:194. doi: 10.1186/s13075-0161092-0

Rahimi, H., Dieudonne, G., Kheyfits, V., Bouta, E. M., Wood, R. W., Barrett, R., et al. (2016b). Relationship between lymph node volume and pain following certolizumab therapy for rheumatoid arthritis flare: a pilot study. Clin. Med. Insights Arthritis Musculoskelet Disord. 9, 203-208. doi: 10.4137/CMAMD. S40237

Rudan, I., Sidhu, S., Papana, A., Meng, S. J., Xin-Wei, Y., Wang, W., et al. (2015). Prevalence of rheumatoid arthritis in low- and middle-income countries: a systematic review and analysis. J. Glob. Health 5:010409. doi: 10.7189/jogh.05. 010409

Scallan, J. P., and Davis, M. J. (2013). Genetic removal of basal nitric oxide enhances contractile activity in isolated murine collecting lymphatic vessels. J. Physiol. 591(Pt 8), 2139-2156. doi: 10.1113/jphysiol.2012.25 0662

Scallan, J. P., Zawieja, S. D., Castorena-Gonzalez, J. A., and Davis, M. J. (2016). Lymphatic pumping: mechanics, mechanisms and malfunction. J. Physiol. 594, 5749-5768. doi: 10.1113/JP272088

Weinblatt, M. E., Keystone, E. C., Furst, D. E., Moreland, L. W., Weisman, M. H., Birbara, C. A., et al. (2003). Adalimumab, a fully human anti-tumor necrosis factor alpha monoclonal antibody, for the treatment of rheumatoid arthritis in patients taking concomitant methotrexate: the ARMADA trial. Arthritis Rheum. 48, 35-45.

Weinblatt, M. E., Kremer, J. M., Bankhurst, A. D., Bulpitt, K. J., Fleischmann, R. M., Fox, R. I., et al. (1999). A trial of etanercept, a recombinant tumor necrosis factor receptor: $F c$ fusion protein, in patients with rheumatoid arthritis receiving methotrexate. N. Engl. J. Med. 340, 253-259.

Zawieja, D. C. (2009). Contractile physiology of lymphatics. Lymphat Res. Biol. 7, 87-96. doi: 10.1089/1rb.2009.0007

Zawieja, S. D., Castorena-Gonzalez, J. A., Dixon, B., and Davis, M. J. (2017). Experimental models used to assess lymphatic contractile function. Lymphat Res. Biol. 15, 331-342. doi: 10.1089/lrb.2017.0052

Zhang, Q., Lu, Y., Proulx, S., Guo, R., Yao, Z., Schwarz, E. M., et al. (2007). Increased lymphangiogenesis in joints of mice with inflammatory arthritis. Arthritis Res. Ther. 9:R118.

Zhou, Q., Wood, R., Schwarz, E. M., Wang, Y. J., and Xing, L. (2010). Near-infrared lymphatic imaging demonstrates the dynamics of lymph flow and lymphangiogenesis during the acute versus chronic phases of arthritis in mice. Arthritis Rheum. 62, 1881-1889. doi: 10.1002/art. 27464

Conflict of Interest: The authors declare that the research was conducted in the absence of any commercial or financial relationships that could be construed as a potential conflict of interest.

Publisher's Note: All claims expressed in this article are solely those of the authors and do not necessarily represent those of their affiliated organizations, or those of the publisher, the editors and the reviewers. Any product that may be evaluated in this article, or claim that may be made by its manufacturer, is not guaranteed or endorsed by the publisher.

Copyright (c) 2021 Scallan, Bouta, Rahimi, Kenney, Ritchlin, Davis and Schwarz. This is an open-access article distributed under the terms of the Creative Commons Attribution License (CC BY). The use, distribution or reproduction in other forums is permitted, provided the original author(s) and the copyright owner(s) are credited and that the original publication in this journal is cited, in accordance with accepted academic practice. No use, distribution or reproduction is permitted which does not comply with these terms. 